Mathematical Modeling

\title{
INTELLIGENT PSYCHO-DIAGNOSTIC FORECASTING SYSTEM
}

\author{
A.N. Danilenko \\ Samara National Research University, Samara, Russia
}

\begin{abstract}
In this article we have considered a problem of psychotherapy efficiency of. We offer a method of assessing and forecasting efficiency of therapeutic influence. The principle of creating intelligence diagnostic forecasting system is described.
\end{abstract}

Keywords: decision support systems, neural networks, fuzzy logic, efficiency of psychotherapy.

Citation: Danilenko AN. Intelligent psycho-diagnostic forecasting system. CEUR Workshop Proceedings, 2016; 1638: 527-535. DOI: $10.18287 / 1613$ 0073-2016-1638-527-535

\section{Introduction}

The main objective of any psychotherapeutic treatment lies in helping patients to introduce necessary changes to their lives. Success or efficiency of psychotherapy is assessed depending on how resistant and, in a broader sense - salutary; these changes are for the patient. Nowadays psychotherapeutic measures providing long-lasting positive effect are considered to be the most suitable. In other words, overall performance of a psychotherapist is evaluated by a result which cannot be subjected to reliable forecasting.

The problem of psychotherapeutic forecast efficiency at the initial stage of treatment has not been solved and the choice of a method which would be adequate to a patient's condition and their personal conditions is carried out by psychotherapists intuitively or on the basis of their professional experience. Therefore, this aspect of psychotherapeutic treatment has a subjective character while the majority of psychotherapeutic models do not even consider measuring dynamics of the patient's condition and assessing treatment efficiency. 


\section{Method of efficiency predictive assessment of psychotherapeutic influence}

We believe that efficiency predictive assessing of psychotherapeutic influence can be based on a series of measurements conducted for indicators of mental and, naturally, emotional condition of the patient. If we exclude diagnostics methods of a functional state by removing psycho-physiological indicators, e.g., questionnaires, aimed at selfassessment of the mental state of examinees we will arrive at the most widespread method of forecasting.

One of the diagnostic methods which would meet the requirements of serial indicators measurement is the color test by M. Lyusher. This method has been chosen by us because it is not bound to lexical or graphic questions and terms which result in the so-called "effect of learning" at repeated tests and seriously distort the results of testing. Long-term applying Lyusher's test (the essence of which is detecting examinee's choice of the preferred and non-preferred colors out of the eight shown colors) demonstrates that color preferences depend on a set of basic personal characteristics as well as on the actual state caused by a particular situation.

The offered method of assessment and forecast of the examinee's condition is based on calculating the described criteria. Since the testing procedure is brief and simple and its contents bear unconscious character, it can be repeated several times, with short time intervals without the loss of results reliability. It provides the opportunity of forecasting patient's condition dynamics by means of trends creating methods.

\section{Intelligent emotional condition dynamic forecasting system}

In order to forecast the change in the examinee's emotional state of we apply the indistinct neural production network by Vanga-Mendel. Let us examine the forecast task in more details in the example below.

Throughout the psychotherapeutic course the patient takes tests with a certain frequency which corresponds to the frequency of carrying out psychotherapeutic sessions (usually not more often than once a week). As a result, the data file with indicators of this patient's emotional condition is being collected. By carrying out each test we will receive the value of seven indicators of an emotional state. These indicators form a complex assessment of an emotional state. For the purpose of maximum saving of the examinee's information we have decided to consider these indicators independently without carrying out a regression generalization. In this regard, the forecast for each indicator becomes irrespective of its own neural network. The forecast of one of the indicators will be described further in this paper.

For the actual forecast to be successful it is necessary to know the values of at least the same number of test results, as the number of the accesses into the neural network. The values of the latest tests are used for the forecast below. While implementing the forecast, the predicted values are used instead of the missing values. Therefore, the process of forecasting becomes interactive. 
For implementing the forecast function in indistinct neural networks we have applied an indistinct neural production network by Vanga-Mendel. It implements the indistinct production model based on rules of the following type:

$$
\begin{gathered}
\text { If } x_{1} \text { there is } A_{i 1} \& \ldots \& x_{j} \text { there is } A_{i j} \& \ldots \& x_{m} \text { there is } A_{i m}, \\
\text { THEN } y \text { there is } B_{i}, i=1, \ldots, n
\end{gathered}
$$

Moreover, the algorithm of an indistinct conclusion which is carried out by this indistinct neural production network is based on the following provisions:

- The accessory functions of all the indistinct sets are represented by a Gauss function;

- Indistinct implication - indistinct work;

- T-norm - indistinct work;

- Accumulation of the active conclusions of rules is not carried out;

- The defusification method - the average center.

Thus, the indistinct production model and the mechanism of an indistinct conclusion for this indistinct neural production network can be displayed by the following expression:

$$
\mu_{B^{\prime}{ }_{i}}(y)=\mu_{B_{i}}(y) \prod_{j=1}^{m} \mu_{A_{i j}}\left(x_{j}^{\prime}\right)
$$

As accumulation of rules is not carried out and the method of a defusification is the method of the average center, a defusified value is determined by the output change in the formula:

$$
y^{\prime}=\frac{\sum_{i=1}^{n}\left(\arg \max _{y} \mu_{B_{i}^{\prime}}(y) \prod_{j=1}^{m} \mu_{A_{i j}}\left(x_{j}^{\prime}\right)\right)}{\sum_{i=1}^{n} \prod_{j=1}^{m} \mu_{A_{i j}}\left(x_{j}^{\prime}\right)}
$$

In our case the accessory functions of all indistinct sets are represented by the Gauss functions. Therefore, expression (2) is put in the following way:

$$
y^{\prime}=\frac{\sum_{i=1}^{n}\left(\arg \max \left(\exp \left[-\frac{y-c_{i}}{d_{i}}\right]\right) \cdot \prod_{j=1}^{m} \exp \left[-\left(\frac{x_{j}^{\prime}-a_{i j}}{b_{i j}}\right)^{2}\right]\right)}{\sum_{i=1}^{n} \prod_{j=1}^{m} \exp \left[-\left(\frac{x_{j}^{\prime}-a_{i j}}{b_{i j}}\right)^{2}\right]}
$$

Where $c_{i}$ and $d_{i}$ respectively are the centers and the width of the Gaussian functions representing the function of accessory indistinct sets $B_{i}$ of the conclusions of rules; $a_{i}$ and $b_{i}$ - respectively are the centers and the width of the Gaussian functions representing the function of accessory indistinct sets $A_{i}$ of the prerequisites of rules. In the final equation we receive: 
$y^{\prime}=\frac{\sum_{i=1}^{n}\left(c_{i} \cdot \prod_{j=1}^{m} \exp \left[-\left(\frac{x_{j}^{\prime}-a_{i j}}{b_{i j}}\right)^{2}\right]\right)}{\sum_{i=1}^{n} \prod_{j=1}^{m} \exp \left[-\left(\frac{x_{j}^{\prime}-a_{i j}}{b_{i j}}\right)^{2}\right]}$

Expression (4) fully describes the procedure of an indistinct conclusion proceeding from the above provisions. In figure 1 we have represented a multi-layered structure of an indistinct network which layers elements realize the corresponding components of this expression.

The structure of this indistinct network consists of four layers.

Layer 1 carries out a fusification of the variables $x_{j}^{\prime}(j=1, \ldots, n)$. Elements of this layer calculate values of the accessory functions $\mu_{A_{i j}}\left(x_{j}^{\prime}\right)$ set by Gaussian functions with parameters $a_{i j \text { and }} b_{i j}$.

Layer 2 in which the number of the elements is equal to the number of rules in the base carries out the aggregation of degrees of prerequisites validity of the corresponding rules.

In layer 3 the first element serves as a layer for activation of the conclusions of rules,

$c_{i}$ according to the values of the degrees of the prerequisites of rules aggregated in the previous layer. The second element of the layer carries out auxiliary calculations for the subsequent defusification of the result.

Layer 4 which consisting of one element carries out a defusification of an output variable.

Parametrical layers of a network are the first and the third layers where the adjusted parameters are $a_{i j}, b_{i j}$ and $c_{i}$.

The indistinct neural production network of Vanga-Mendel has a multi-layered structure with direct distribution of a signal, the ouput value which can be changed, by correcting parameters of the elements of the layers that allow using the algorithm of the return distribution of a mistake for training the network.

In the set training selection $\left(x_{1}^{(k)}, x_{2}^{(k)}, \ldots, x_{m}^{(k)}, y^{(k)}\right), k=1, \ldots, K, x_{1}^{(k)}, x_{2}^{(k)}, x_{m}^{(k)}$ are the values of accessing variables $x_{1}, x_{2}, \ldots, x_{m}, y^{(k)}$ is the reference value of an output variable $y_{\text {in }} k$.

We will consider procedure of the correction of values, $c_{i}, a_{i j}$ and $b_{i j}$.

Stage 1. For each example of the training selection of values of input variables $\left(x_{1}^{(k)}, x_{2}^{(k)}, \ldots, x_{m}^{(k)}, y^{(k)}\right), k=1, \ldots, K$ values of an output variable, $y^{\prime(k)}$, are calculated.

Stage 2. Function of a mistake for all examples of the training selection is calculated:

$E^{(k)}=\frac{1}{2}\left(y^{\prime(k)}-y^{(k)}\right)^{2}, k=1, \ldots, K$ 
Stage 3. Values $c_{i}, a_{i j}$ and $b_{i j}$ are corrected for every $i$ provided that every example $k$ of the training selection, proceeds from the ratios:

$$
\begin{aligned}
& c_{i}(t+1)=c_{i}(t)-\eta \cdot \frac{\left(y^{\prime(k)}-y^{(k)}\right) \prod_{j=1}^{m} \exp \left[-\left(\frac{x_{j}^{\prime(k)}-a_{i j}}{b_{i j}}\right)^{2}\right]}{\sum_{i=1}^{n} \prod_{j=1}^{m} \exp \left[-\left(\frac{x_{j}^{(k)}-a_{i j}}{b_{i j}}\right)^{2}\right]} \\
& a_{i j}(t+1)=a_{i j}(t)-\eta \cdot \frac{2 \cdot\left(x_{j}^{(k)}-a_{i j}\right) \cdot\left(y^{(k)}-y^{(k)}\right) \cdot\left(c_{i}-y^{(k)}\right) \cdot \prod_{j=1}^{m} \exp \left[-\left(\frac{x_{j}^{\prime(k)}-a_{i j}}{b}\right)^{2}\right]}{b_{i j}^{2} \cdot \sum_{i=1}^{n} \prod_{j=1}^{m} \exp \left[-\left(\frac{x_{j}^{\prime(k)}-a_{i j}}{b_{i j}}\right)^{2}\right]} \\
& b_{i j}(t+1)=b_{i j}(t)-\eta \cdot \frac{2 \cdot\left(x_{j}^{(k)}-a_{i j}\right)^{2} \cdot\left(y^{(k)}-y^{(k)}\right) \cdot\left(c_{i}-y^{(k)}\right) \cdot \prod_{j=1}^{m} \exp \left[-\left(\frac{x_{j}^{(k)}-a_{i j}}{b}\right)^{2}\right]}{b_{i j}^{2} \cdot \sum_{i=1}^{n} \prod_{j=1}^{m} \exp \left[-\left(\frac{x_{j}^{(k)}-a_{i j}}{b_{i j}}\right)^{2}\right]}
\end{aligned}
$$

Where $t$ is the number of training iterations; $\eta \in[0,1]$ is the training speed coefficient.

Stage 4. Values of an output variable for each example of selection $y^{\prime(k)}$ are repeatedly calculated and the average total error, taking into account all examples of the training selection, is calculated and compared to some established threshold:

$$
E=\frac{1}{K} \sum_{k=1}^{K}\left(y^{(k)}-y^{(k)}\right)^{2}<\varepsilon
$$

In the case of a successful performance of a condition the network is considered to be correctly trained. Otherwise, transition to stage 3 is carried out and adjustment repeats.

Functionality of the automated system of the forecast of dynamics of an emotional condition of the personality includes the indicators forecasting function of an emotional condition of the person. To comprehend this function an indistinct neuralproduction network by Vanga-Mendel is used.

Effective functioning of a neural network requires an empirical selection of some parameters, defining structure and networks, and parameters of the algorithm of its training. The forecast window (i.e. a number of accesses into a neural network) is defined in a system development task. The parameters of a neural network available for control are a training coefficient.

To check the working capacity and the selection of necessary parameters the function below is used:

$$
\begin{aligned}
& \mathrm{f}(\mathrm{x})=0.1 \cdot \sin (0.02 \cdot \pi \mathrm{x})+0.2 \cdot \sin (0.03 \cdot \pi \mathrm{x})+0.6 \cdot \sin (0.9 \cdot \pi \mathrm{x})+ \\
& +1.11 \cdot \sin (0.19 \cdot \pi \mathrm{x})+2.3 \cdot \sin (0.37 \cdot \pi \mathrm{x})
\end{aligned}
$$

Its schedule and results of the forecast for a neural network are represented in figure 1 . 
In the function highlighted in blue, on the basis of which training was made, the red color corresponds to the values predicted by a neural network after training. Highlighted green portion of the area of the function has also been used for training. Other area has been used as a test selection for determining the forecast quality of the unknown data. Training was made before achieving the average total error training (formula 4) of 0.01 .

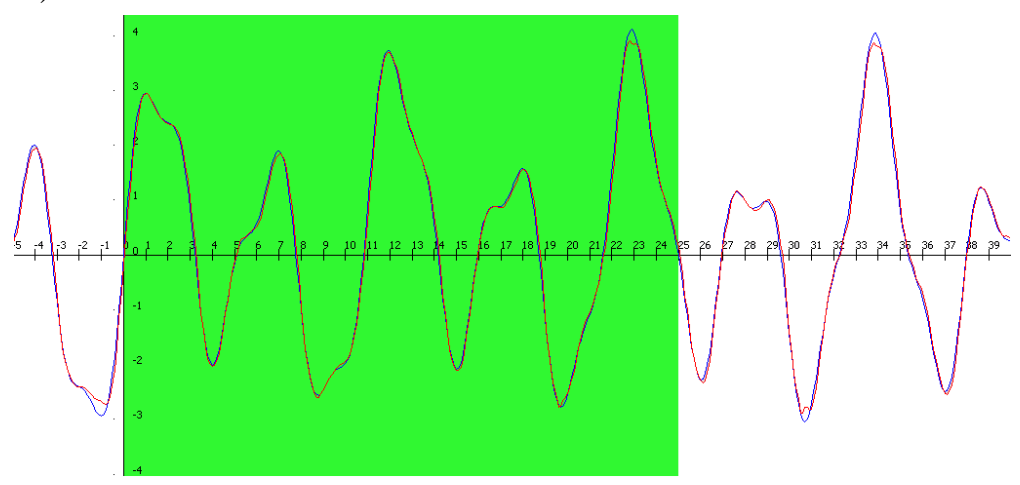

Fig. 1. Function graph of testing and results of the forecast

For the selection of the training coefficient value, a study of dependence of the number of iterations to achieve an error of training units (training speed) and errors on test selection from the training coefficient value has been conducted at 0.01 . Results are given in table 1.

Table 1. Selection of the training coefficient $\mathrm{f}$

\begin{tabular}{ccc}
\hline Training coefficient & Number of iterations & Error \\
\hline $\mathbf{0 . 1}$ & 319 & 0.0126977 \\
$\mathbf{0 . 2}$ & 154 & 0.0115617 \\
$\mathbf{0 . 3}$ & 113 & 0.0122632 \\
$\mathbf{0 . 4}$ & 87 & 0.0126165 \\
$\mathbf{0 . 5}$ & 73 & 0.0120938 \\
$\mathbf{0 . 6}$ & 63 & 0.0117784 \\
$\mathbf{0 . 7}$ & 61 & 0.0116801 \\
$\mathbf{0 . 8}$ & 60 & 0.0134587 \\
$\mathbf{0 . 9}$ & 56 & 0.0122873 \\
\hline
\end{tabular}

The graph on which the value of an error on test has reached 10,000 for descriptive reasons is given in figure 2 .

It is evident from the graph that the number of iterations considerably decreases as the coefficient value of training increases. Thus, the error slightly increases at great coefficient values of training. Also if the values of the training coefficient are too large the instability of work of the algorithm of training is possible. Therefore, it is expedient to choose coefficient of training equal 0.7 . 
For studying the quality of the forecast, research of dependence of an error upon test selection from number of steps of the forecast has been conducted. Results are presented in table 2 .

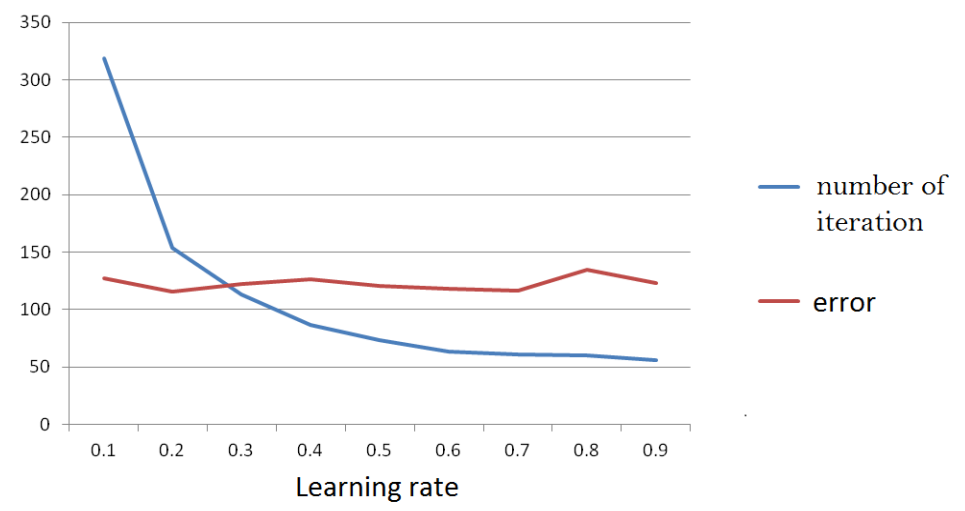

Fig. 2. Selection of the training coefficient

Table 2. Selection of the training coefficient

\begin{tabular}{cc}
\hline Number of steps of the forecast & Error \\
\hline $\mathbf{1}$ & 0.004398821 \\
$\mathbf{2}$ & 0.017341442 \\
$\mathbf{3}$ & 0.059260252 \\
\hline
\end{tabular}

Training was conducted until an error of 0.02 was achieved on the training selection. It is evident from the table that the transition from the forecast for step one to the forecast for step two the error increases by 4 times, upon transition to step three -14 times. Taking into account that the network was trained for the forecast for one step and the main objective is the forecast for one step, these results can be considered satisfactory, since an error even at the forecast for step 3 keeps the same order as at the forecast for step 1.

\section{A control variable}

For a control variable tests to be taken by several people were held to collect data for training a neural network. The structure of a control variable included taking the test, viewing the results of the test and analyzing the graph of change of indicators of an emotional state with the forecast.

The testee is offered to choose the color which is mostly preferred by them by pressing on the desired selection. After the choice is made, the chosen color is removed from the choice panel. After all colors are eslected, a message on a successful completion of the test is demonstrated to the testee. 
In figure 4 the graph displays the results of choices one after another, a standard color, a code for a standard color, the corresponding tags, stress and alarm tags. In a separate field, the psychologist can leave comments to this test in the form of a saved text.

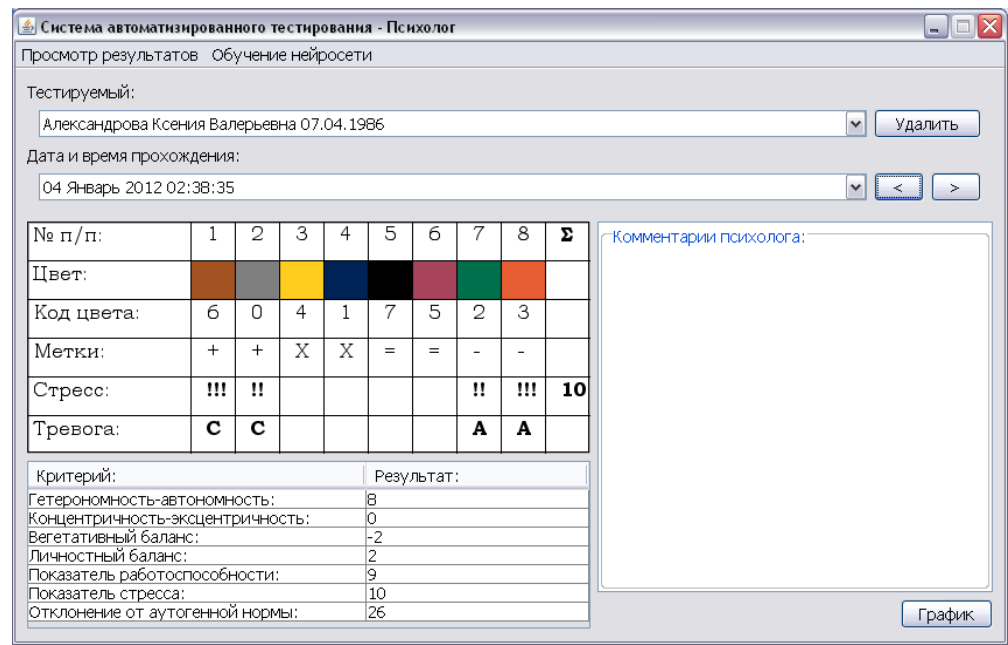

Fig. 3. Main window of the psychologist, browse mode of results

In order to view the graph of dynamics of emotional indicators, it is necessary to press 'Graph' button. Then there will pop-up a corresponding window (figure 5). In the left part of the graph the log of changes of the chosen indicator is represented. In the right part, green color indicates the forecast of change of this indicator for three steps.

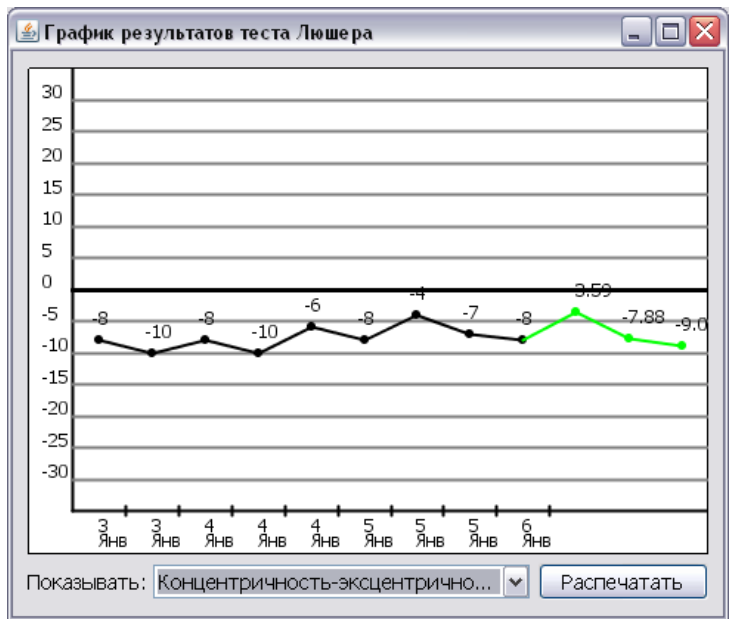

Fig. 4. Graph of changes of emotional indicators

For passing a control test, basic training of a neural network on the given tests of the three testees has been provided. The total number of tests was 19, 16 of them had been taken by other testees in the training selection, 3 - in the test mode. 


\section{Conclusion}

The proposed method shows the real possibility of designing an intelligent system to estimate and to predict the psychotherapeutic influence at early stages of interaction between a psychotherapist and a patient. The method based on the classic psychodiagnostic procedures is easy to use by the patients and therapists.

\section{References}

1. Wallerstein R, Robbins L, Sargent H, Luborsky L. The psychotherapy Research Project of the Menninger Foundation: Rationale, Method and Sample Use. First Report. Bull. of Menninger Clin., 1956; 20: 221-278.

2. Nemchin TA. Conditions of psychological tension. L., 1983.

3. Hofler M, Gloster AT, Hoyer J. Causal effects in psychotherapy: Counterfactuals counteract overgeneralization. Psychotherapy Research, 2010.

4. Kazdin A. Methodology, design, and evaluation in psychotherapy research. Handbook of psychotherapy and behavioral change. 4th ed., 1994: 19-71.

5. Spence D. Traditional case studies and prescriptions for improving them. Psychodynamic treatment research. A handbook for clinical practice. Wiley, 1993.

6. Luborsky L, Crits-Christoph P, Mintz J, Auerbach A. Who Will Benefit From Psychotherapy. N.Y.: Basic Books, 1988.

7. Spirtes P, Glymour CN, Scheines R. Causation, Prediction, and Search. Springer-Verlag, New York, 1993.

8. Zinchenko EA. Metod of expert visual definition of working emotional conditions on production, 1983: 59-63.

9. Karmanov AA. Methodology of diagnostics of key parameters of a mental state in Lyusher's test. URL: http://koob.ru.

10. Prokhorov AO. Mental states and their manifestations in educational process. Kazan, 1991.

11. Sobchik LN. Metod of color elections - modification of eight-color test of Lyusher. Practical guidance. $\mathrm{SPb} .:$ Speech, 2010; 128 p.

12. Manski C. Identification for Prediction and Decision. Harvard University Press, Cambridge, Massachusetts, 2007.

13. Bareinboim E, Brito C, Pearl J. Local characterizations of causal bayesian networks. Lecture Notes in Artificial Intelligence, 2012; 7205: 1-17.

14. Pearl J. The deductive approach to causal inference. Journal of Causal Inference, 2014; 2(2): 115-130.

15. Osovsky S. Neural networks for information processing. Finance and Statistics, 2002; 344 p.

16. Borisov VV, Kruglov VV, Fedulov AS. Indistinct models and networks. The hot line Telecom, 2007; 284 p.

17. Didelez V, Kreiner S, Keiding N. Graphical models for inference under outcomedependent sampling. Statistical Science, 2010; 25(3): 368-387.

18. Bareinboim E, Pearl J. A general algorithm for deciding transportability of experimental results. Journal of Causal Inference, 2013; 1(1): 107-134.

19. Bareinboim E, Pearl J. Controlling selection bias in causal inference. In Proceedings of the 15th International Conference on Artificial Intelligence and Statistics (AISTATS), JMLR, April 21-23, 2012: 100-108. 